\title{
Research Article \\ Critical Oscillation Constant for Difference Equations with Almost Periodic Coefficients
}

\author{
Petr Hasil ${ }^{1}$ and Michal Veselý ${ }^{2}$ \\ ${ }^{1}$ Department of Mathematics, Mendel University in Brno, Zemědělská 1, 61300 Brno, Czech Republic \\ ${ }^{2}$ Department of Mathematics and Statistics, Masaryk University, Kotlářská 2, 61137 Brno, Czech Republic
}

Correspondence should be addressed to Petr Hasil, hasil@mendelu.cz

Received 28 May 2012; Revised 10 September 2012; Accepted 13 September 2012

Academic Editor: Elena Braverman

Copyright (c) 2012 P. Hasil and M. Veselý. This is an open access article distributed under the Creative Commons Attribution License, which permits unrestricted use, distribution, and reproduction in any medium, provided the original work is properly cited.

We investigate a type of the Sturm-Liouville difference equations with almost periodic coefficients. We prove that there exists a constant, which is the borderline between the oscillation and the nonoscillation of these equations. We compute this oscillation constant explicitly. If the almost periodic coefficients are replaced by constants, our result reduces to the well-known result about the discrete Euler equation.

\section{Introduction}

In this paper, we analyse the second-order Sturm-Liouville equation

$$
\Delta\left(r_{k} \Delta y_{k}\right)+q_{k} y_{k+1}=0, \quad r_{k}>0
$$

whose oscillation properties are widely studied over the last few decades. We begin with a short literature overview concerning the (non)oscillation of $(*)$ and of some direct generalizations (including half-linear equations and dynamic equations on time scales).

Basic necessary and sufficient conditions for $(*)$ in order to be oscillatory are derived in [1-3]. In [4] (see also [5]), the concept of a phase is established to obtain other oscillation criteria. For the matrix difference equations of the form of $(*)$, we refer to [6]. Several oscillation criteria for slightly more general equations are presented in $[7,8]$. The oscillation theory for the corresponding higher-order two-term Sturm-Liouville difference equations can be found in [9-11] (for differential case, see [12]).

Fundamental aspects of Sturmian theory (and some oscillation criteria) for secondorder Sturm-Liouville equations on arbitrary time scales are formulated in [13]. Oscillation 
criteria for second-order difference equations can be obtained from oscillation criteria for more general dynamic equations. The oscillation properties of second-order linear dynamic equations, which have the Sturm-Liouville difference equations as special cases, are considered, for example, in [14].

As an illustration, we mention a particular Sturm-Liouville equation of which the complete oscillation classification is done as a consequence of general results on time scales. Using the comparison theorem for second-order linear dynamic equations, it is shown in [15] that the difference equation

$$
\Delta^{2} y_{k}+b \frac{(-1)^{k}}{k^{c}} y_{k+1}=0
$$

is oscillatory for any $c<1$ and $b \neq 0$. Further, it is obtained in [16] (based on results of $[17,18]$ ) that the equation

$$
\Delta^{2} y_{k}+\left[\frac{a}{k^{2}}+b \frac{(-1)^{k}}{k}\right] y_{k+1}=0
$$

is oscillatory if and only if $4 a>1-b^{2}$. Finally in [16], applying the Willett-Wong-type theorems for second-order linear dynamic equations, there is given the full oscillation analysis of $(*)$ for

$$
r_{k}=1, \quad q_{k}=\frac{a}{k^{c+1}}+b \frac{(-1)^{k}}{k^{c}}
$$

with regard to arbitrary $a, b, c \in \mathbb{R}$.

The importance of the oscillation results about second-order equations lies among others in the fact that such results can be used to study the oscillation and nonoscillation properties of solutions of different equations. For example (see [19] and also [20]), all solutions of the delay equation $\Delta y_{k}+p_{k} y_{k-n}=0$ oscillate if and only if all solutions of a certain type of $(*)$ with $r_{k}=1$ oscillate.

The main aim of this paper is to present a sharp oscillation constant for the Euler-type difference equation

$$
\Delta\left(r_{k} \Delta y_{k}\right)+\frac{r s_{k}}{(k+1) k} y_{k+1}=0
$$

where $\gamma \in \mathbb{R}, \inf \left\{r_{k}\right\}>0$, and $\left\{r_{k}\right\},\left\{s_{k}\right\}$ are positive almost periodic sequences. More precisely, we show that $(* *)$ is the so-called conditionally oscillatory; that is, we prove that there exists a positive constant $K$ (the oscillation constant) such that $(* *)$ is oscillatory for $r>K$ and non-oscillatory for $\gamma<K$.

Our research is motivated by the continuous case. It is a famous result due to Kneser [21] that the differential Euler equation

$$
y^{\prime \prime}(t)+\frac{\gamma}{t^{2}} y(t)=0
$$


is conditionally oscillatory with the oscillation constant $K=1 / 4$. It is known (see [22]) that the equation

$$
\left[r(t) y^{\prime}(t)\right]^{\prime}+\frac{r s(t)}{t^{2}} y(t)=0
$$

where $r, s$ are positive periodic continuous functions, is conditionally oscillatory as well. We also refer to [23] and [24-29] which generalize [23] (for the discrete case, see [30]). Since the Euler difference equation

$$
\Delta^{2} y_{k}+\frac{r}{(k+1) k} y_{k+1}=0
$$

is conditionally oscillatory with the oscillation constant $K=1 / 4$ (see [31]), it is natural to analyse the conditional oscillation of $(* *)$. Note that the announced result is more general than the results known in the continuous case, because $(* *)$ has almost periodic coefficients. The conditional oscillation of discrete equations with constant coefficients can be generalized in other ways. Point out [32], where an oscillation constant is characterized. The constant from [32] coincides with our oscillation constant if the considered coefficients are asymptotically constant.

Solutions of the second-order Sturm-Liouville difference equations with periodic coefficients are studied in [33] (see also [34,35]). In [36], the half-linear differential equations of the second order with the Besicovitch almost periodic coefficients are considered and an oscillation theorem for these equations is obtained.

In the last years, many results dealing with the conditional oscillation of second-order equations and two-term equations of even order appeared. The two-term difference equation of even order

$$
(-1)^{n+1} \Delta^{n}\left(\frac{\Gamma(k+1)}{\Gamma(k-\alpha+1)} \Delta^{n} y_{k}\right)+q_{k} y_{k+n}=0
$$

where $\Gamma$ denotes the gamma function, is studied in $[9,10]$. Results concerning the half-linear difference equation

$$
\Delta\left[r_{k} \Phi\left(\Delta y_{k}\right)\right]+q_{k} \Phi\left(y_{k+1}\right)=0
$$

where

$$
r_{k}>0, \quad \Phi(y)=|y|^{\alpha-1} \operatorname{sgn} y, \quad \alpha>1,
$$

can be found in [37] for $r_{k}=1, q_{k}=\gamma(k+1)^{-\alpha}$ and also in [38, 39] (for dynamic half-linear equations on time scales, see [40-42]).

The paper is organized as follows. In Section 2, we mention only necessary preliminaries and an auxiliary result. Our main result is proved in Section 3, where the particular case concerning the equation with periodic coefficients is formulated as well. The paper is finished by concluding remarks and simple examples. 


\section{Preliminaries}

We begin this section recalling some elements of the oscillation theory of the Sturm-Liouville difference equation

$$
\Delta\left(r_{k} \Delta y_{k}\right)+q_{k} y_{k+1}=0, \quad r_{k}>0, k \in \mathbb{N}
$$

For more details, we can refer to books $[43,44]$ and references cited therein.

We recall that an interval $(a, a+1], a \in \mathbb{N}$, contains the generalized zero of a solution $\left\{y_{k}\right\}$ of (2.1) if $y_{a} \neq 0$ and $y_{a} y_{a+1} \leq 0$. Equation (2.1) is said to be conjugate on $\{a, \ldots, a+n\}$, $n \in \mathbb{N}$, if there exists a solution which has at least two generalized zeros on $(a, \ldots, a+n+1]$ or if the solution $\left\{\tilde{y}_{k}\right\}$ satisfying $\tilde{y}_{a}=0$ has at least one generalized zero on $(a, \ldots, a+n+1]$. Otherwise, (2.1) is said to be disconjugate on $\{a, \ldots, a+n\}$. Since Sturmian theory is valid for difference equations, all solutions of (2.1) have either a finite or an infinite number of generalized zeros on $\mathbb{N}$. Hence, we can categorize these equations as oscillatory and nonoscillatory.

Definition 2.1. Equation (2.1) is called non-oscillatory provided a solution of (2.1) is disconjugate at infinity, that is, there exists $N \in \mathbb{N}$ such that (2.1) is disconjugate on any set $[N, N+m] \cap \mathbb{N}, m \in \mathbb{N}$. Otherwise, we say that (2.1) is oscillatory.

Since we study a special case of (2.1), when the coefficients are almost periodic, we also mention the basics of the theory of almost periodic sequences. Here, we refer to each one of books $[45,46]$.

Definition 2.2. A real sequence $\left\{f_{k}\right\}_{k \in \mathbb{Z}}$ is called almost periodic if, for any $\varepsilon>0$, there exists a positive integer $p(\varepsilon)$ such that any set consisting of $p(\varepsilon)$ consecutive integers contains at least one integer $l$ with the property that

$$
\left|f_{k+l}-f_{k}\right|<\varepsilon, \quad k \in \mathbb{Z}
$$

We say that a sequence $\left\{g_{k}\right\}_{k=1}^{\infty}$ is almost periodic if there exists an almost periodic sequence $\left\{f_{k}\right\}_{k \in \mathbb{Z}}$ for which $f_{k}=g_{k}, k \in \mathbb{N}$.

The above definition of almost periodicity is based on the Bohr concept. Now we formulate a necessary and sufficient condition for a sequence to be almost periodic. The following theorem is often used as an equivalent definition (the Bochner one) of almost periodicity for $k \in \mathbb{Z}$.

Theorem 2.3. A sequence $\left\{f_{k}\right\}_{k \in \mathbb{Z}} \subset \mathbb{R}$ is almost periodic if and only if any sequence of the form $\left\{f_{k+h(n)}\right\}$, where $h(n) \in \mathbb{Z}, n \in \mathbb{N}$, has a uniformly convergent subsequence with respect to $k$.

Proof. See [45, Theorem 1.26].

Corollary 2.4. Let $\left\{f_{k}\right\}$ be almost periodic. The sequence $\left\{1 / f_{k}\right\}$ is almost periodic if and only if

$$
\inf \left\{\left|f_{k}\right| ; k \in \mathbb{N}\right\}>0 .
$$


Proof. The corollary follows from [45, Theorem 1.27] and [47, Theorem 1.9] (or directly from Theorem 2.3). It suffices to use that (2.3) implies $\inf \left\{\left|f_{k}\right|\right\}>0$ for any almost periodic sequence $\left\{f_{k}\right\}$ if $k \in \mathbb{Z}$.

Note that there exist nonzero almost periodic sequences $\left\{f_{k}\right\}$ for which (2.3) is not satisfied (see, e.g., [48, Theorem 3]).

Theorem 2.5. If $\left\{f_{k}\right\}$ is an almost periodic sequence, then the limit

$$
M\left(\left\{f_{k}\right\}\right):=\lim _{n \rightarrow \infty} \frac{f_{k}+f_{k+1}+\cdots+f_{k+n}}{n+1}
$$

exists uniformly with respect to $k$.

Proof. See [45, Theorem 1.28].

Definition 2.6. Let $\left\{f_{k}\right\}$ be almost periodic. The number $M\left(\left\{f_{k}\right\}\right)$ introduced in (2.4) is called the mean value of $\left\{f_{k}\right\}$.

Remark 2.7. For any positive almost periodic sequence $\left\{f_{k}\right\}$, we have $M\left(\left\{f_{k}\right\}\right)>0$. Indeed, if we put $\varepsilon=f_{1} / 2$ and find a corresponding $p(\varepsilon)$ in Definition 2.2, then we obtain

$$
M\left(\left\{f_{k}\right\}\right) \geq \frac{f_{1}}{2 p(\varepsilon)}>0
$$

In the proof of our main result, we use an adapted Riccati technique. The classical Riccati technique deals with the so-called Riccati difference equation, which we obtain from (2.1) using the substitution $w_{k}=r_{k}\left(\Delta y_{k} / y_{k}\right)$, that is, we obtain the equation

$$
\Delta w_{k}+q_{k}+\frac{w_{k}^{2}}{w_{k}+r_{k}}=0, \quad k \in \mathbb{N}
$$

Putting $\zeta_{k}=-k w_{k}$, we adapt (2.6) to our purposes. A direct calculation leads to the equation

$$
\Delta \zeta_{k}=\frac{1}{k}\left[k(k+1) q_{k}+\zeta_{k}+\frac{(k+1) \zeta_{k}^{2}}{k r_{k}-\zeta_{k}}\right], \quad k \in \mathbb{N} .
$$

We also mention two lemmas which we use to prove the main result.

Lemma 2.8. Let the equation

$$
\Delta\left(r_{k} \Delta y_{k}\right)+q_{k} y_{k+1}=0, \quad k \in \mathbb{N},
$$

where $\sup \left\{r_{k} ; k \in \mathbb{N}\right\}<\infty$ and $r_{k}, q_{k}>0, k \in \mathbb{N}$, be non-oscillatory. For any solution $\left\{\zeta_{k}\right\}$ of the associated equation (2.7), there exists $k_{0} \in \mathbb{N}$ such that, if $\zeta_{k_{0}+m}<0$ for some $m \in \mathbb{N}$, then $\zeta_{k+m}<0$, $k \geq k_{0}$. 
Proof. Let $\left\{y_{k}\right\}$ be a solution of the non-oscillatory equation (2.8) for which $y_{k} y_{k+1}>0, k \geq$ $k_{0}$. From [43, Lemma 6.6.1] it follows that the sequence $\left\{w_{k}\right\}_{k=k_{0}}^{\infty}$, where $w_{k}=r_{k}\left(\Delta y_{k} / y_{k}\right)$, is decreasing. Further, [43, Theorem 6.6.2] implies that $\lim _{k \rightarrow \infty} w_{k}=0$. Thus, the sequence $\left\{w_{k}\right\}_{k=k_{0}}^{\infty}$ is positive, that is, $\zeta_{k}=-k w_{k}<0, k \geq k_{0}$.

Lemma 2.9. If there exists a solution $\left\{\zeta_{k}\right\}$ of the associated equation (2.7) satisfying $\zeta_{k}<0$ for all $k \geq k_{0}$, then (2.8) is non-oscillatory.

Proof. The statement of the lemma follows from [44, Theorem 6.16].

\section{Oscillation Constant}

This section is devoted to the main result of our paper. After its proof, within the concluding remarks, we formulate as a corollary the result which deals with periodic equations. This corollary is the discrete counterpart of the main result of [49].

Theorem 3.1. Let the equation

$$
\Delta\left(r_{k} \Delta x_{k}\right)+\frac{r s_{k}}{(k+1) k} x_{k+1}=0, \quad k \in \mathbb{N},
$$

where $\gamma \in \mathbb{R}$ and $\left\{r_{k}\right\}$ and $\left\{s_{k}\right\}$ are positive almost periodic sequences satisfying

$$
\inf \left\{r_{k} ; k \in \mathbb{N}\right\}>0,
$$

be arbitrarily given. Let

$$
K:=\left[4 M\left(\left\{r_{k}^{-1}\right\}\right) M\left(\left\{s_{k}\right\}\right)\right]^{-1}
$$

Then, (3.1) is oscillatory for $\gamma>K$ and non-oscillatory for $\gamma<K$.

Proof. At first, let us prepare several estimates which we will use to prove the theorem. Henceforth, for given $\gamma \neq K$, we will consider $\alpha \in \mathbb{N}$ and $\vartheta>0$ such that

$$
\begin{gathered}
\frac{1}{\alpha} \sum_{i=k}^{k+\alpha-1} s_{i}>2 \vartheta, \quad k \in \mathbb{N}, \\
8\left|\frac{1}{\alpha} \sum_{i=k}^{k+\alpha-1} \frac{1}{r_{i}}-\frac{1}{\alpha} \sum_{i=l}^{l+\alpha-1} \frac{1}{r_{i}}\right|<\left(\frac{1}{\alpha} \sum_{i=1}^{\alpha} \frac{1}{r_{i}}\right)^{2}|\gamma-K| \vartheta, \quad k, l \in \mathbb{N}, \\
2\left|K-\left(\frac{4}{\alpha^{2}} \sum_{i=k}^{k+\alpha-1} \frac{1}{r_{i}} \sum_{i=l}^{l+\alpha-1} s_{i}\right)^{-1}\right|<|\gamma-K|, \quad k, l \in \mathbb{N} .
\end{gathered}
$$


The fact that such numbers $\alpha, \vartheta$ exist follows from Theorem 2.5 and Remark 2.7 (consider also Corollary 2.4 with (3.2)). We put

$$
r^{-}:=\inf \left\{r_{k} ; k \in \mathbb{N}\right\}, \quad r^{+}:=\sup \left\{r_{k} ; k \in \mathbb{N}\right\}, \quad s^{+}:=\sup \left\{s_{k} ; k \in \mathbb{N}\right\}
$$

The adapted Riccati equation associated to (3.1) has the form (see (2.7))

$$
\Delta \zeta_{k}=\frac{1}{k}\left[r s_{k}+\zeta_{k}+\frac{(k+1) \zeta_{k}^{2}}{k r_{k}-\zeta_{k}}\right]
$$

Since one can express

$$
\zeta_{k}+\frac{(k+1) \zeta_{k}^{2}}{k r_{k}-\zeta_{k}}=\frac{k \zeta_{k}\left(r_{k}+\zeta_{k}\right)}{k r_{k}+\left|\zeta_{k}\right|} \quad \text { if } \zeta_{k}<0 \text { for some } k \in \mathbb{N}
$$

it is valid that

$$
\Delta \zeta_{k}>\frac{r s_{k}}{k}>0 \text { if } \zeta_{k}<-r^{+} \text {for some } k \in \mathbb{N},
$$

and that $(C>0$ is arbitrarily given)

$$
\begin{aligned}
\left|\Delta \zeta_{k}\right| & \leq \frac{1}{k}\left[r s^{+}+\frac{k\left|\zeta_{k}\right|\left(r_{k}+\left|\zeta_{k}\right|\right)}{k r_{k}+\left|\zeta_{k}\right|}\right] \\
& <\frac{r s^{+} r^{-}+C\left(r^{+}+C\right)}{k r^{-}} \text {if } \zeta_{k} \in(-C, 0) \text { for some } k \in \mathbb{N}
\end{aligned}
$$

Particularly, if $\zeta_{k}<0$ for all sufficiently large $k$, then there exists $\delta<0$ such that

$$
\zeta_{k}>\delta \text { for considered } k
$$

Indeed, it follows directly from (3.10) and (3.11).

Similarly, applying (3.10) and (3.11), it is seen that there exists $\bar{k}=\bar{k}(\delta, \alpha) \in \mathbb{N}$ for any $\delta<0$ and $\alpha \in \mathbb{N}$ with the property that the solution $\left\{\zeta_{k}\right\}_{k=\bar{k}+m}^{\infty}$ of the Cauchy problem

$$
\Delta \zeta_{k}=\frac{1}{k}\left[\gamma s_{k}+\zeta_{k}+\frac{(k+1) \zeta_{k}^{2}}{k r_{k}-\zeta_{k}}\right], \quad \zeta_{\bar{k}+m}=\zeta_{0} \in(2 \delta, \delta],
$$

where $m \in \mathbb{N}$, satisfies

$$
\left\{\zeta_{k}\right\}_{k=\bar{k}+m}^{\infty} \subseteq\left(\min \left\{2 \zeta_{0},-2 r^{+}\right\}, \infty\right), \quad\left\{\zeta_{k}\right\}_{k=\bar{k}+m}^{\bar{k}+m+\alpha-1} \subseteq\left(\min \left\{2 \zeta_{0},-2 r^{+}\right\}, 0\right),
$$


and hence there exists $\Theta>0$ (consider again (3.11)) for which

$$
\left|\zeta_{\bar{k}+m}-\zeta_{\bar{k}+m+j}\right|<\frac{\Theta}{\bar{k}}, \quad j \in\{1, \ldots, \alpha-1\}, m \in \mathbb{N}
$$

Now we can proceed to the oscillatory part of the theorem. By contradiction, we suppose that $\gamma>K$ and that (3.1) is non-oscillatory. According to Lemma 2.8, any solution $\left\{\zeta_{k}\right\}_{k=k_{0}}^{\infty}$ of the associated adapted Riccati equation (3.8) for which $\zeta_{k_{0}}<0$ satisfies $\zeta_{k}<0$, $k \geq k_{0}$, if $k_{0}$ is enough large. Further, (3.12) gives the existence of $\delta<0$ with the property that $\zeta_{k} \in(\delta, 0)$ for all $k \geq k_{0}$. Using (3.11), we obtain

$$
\left|\Delta \zeta_{k}\right|<\frac{r s^{+} r^{-}-\delta\left(r^{+}-\delta\right)}{k r^{-}}, \quad k \geq k_{0}
$$

Our goal is to achieve a contradiction with $\zeta_{k} \in(\delta, 0)$ by estimating the arithmetic mean of $\alpha$ subsequent values of $\zeta_{k}$. We denote

$$
\xi_{k}:=\frac{1}{\alpha} \sum_{i=k}^{k+\alpha-1} \zeta_{i} \in(\delta, 0), \quad k \geq k_{0}
$$

and compute (for $\left.\Delta \xi_{k}>0\right)$

$$
\begin{aligned}
\Delta \xi_{k} & =\frac{1}{\alpha} \sum_{i=k}^{k+\alpha-1} \Delta \zeta_{i}=\frac{1}{\alpha} \sum_{i=k}^{k+\alpha-1} \frac{1}{i}\left[\gamma s_{i}+\zeta_{i}+\frac{(i+1) \zeta_{i}^{2}}{i r_{i}-\zeta_{i}}\right] \\
& \geq \frac{1}{k+\alpha-1}\left[\frac{\gamma^{k+\alpha-1}}{\alpha} \sum_{i=k}^{k} s_{i}+\xi_{k}+\frac{1}{\alpha} \sum_{i=k}^{k+\alpha-1} \frac{(i+1) \zeta_{i}^{2}}{i r_{i}-\zeta_{i}}\right] \\
& =\frac{1}{k+\alpha-1}\left\{\frac{\gamma^{k+\alpha-1}}{\alpha} \sum_{i=k}^{k} s_{i}-\frac{A_{k}^{2}}{2}+\frac{1}{\alpha} \sum_{i=k}^{k+\alpha-1}\left[\frac{(i+1) \zeta_{i}^{2}}{i r_{i}-\zeta_{i}}-\frac{\zeta_{i}^{2}}{r_{i}}\right]+\xi_{k}+\frac{A_{k}^{2}}{2}+\frac{B_{k}^{2}}{2}+\frac{1}{\alpha} \sum_{i=k}^{k+\alpha-1} \frac{\zeta_{i}^{2}}{r_{i}}-\frac{B_{k}^{2}}{2}\right\}
\end{aligned}
$$

or $\left(\right.$ for $\left.\Delta \xi_{k}<0\right)$

$$
\begin{gathered}
\Delta \xi_{k}=\frac{1}{\alpha} \sum_{i=k}^{k+\alpha-1} \Delta \zeta_{i} \geq \frac{1}{k}\left\{\frac{r^{k}}{\alpha} \sum_{i=k}^{k+\alpha-1} s_{i}-\frac{A_{k}^{2}}{2}+\frac{1}{\alpha} \sum_{i=k}^{k+\alpha-1}\left[\frac{(i+1) \zeta_{i}^{2}}{i r_{i}-\zeta_{i}}-\frac{\zeta_{i}^{2}}{r_{i}}\right]\right. \\
\left.+\xi_{k}+\frac{A_{k}^{2}}{2}+\frac{B_{k}^{2}}{2}+\frac{1}{\alpha} \sum_{i=k}^{k+\alpha-1} \frac{\zeta_{i}^{2}}{r_{i}}-\frac{B_{k}^{2}}{2}\right\},
\end{gathered}
$$

where

$$
A_{k}:=\left(\frac{2}{\alpha} \sum_{i=k}^{k+\alpha-1} \frac{1}{r_{i}}\right)^{-1 / 2}, \quad B_{k}:=\left|\xi_{k}\right|\left(\frac{2}{\alpha} \sum_{i=k}^{k+\alpha-1} \frac{1}{r_{i}}\right)^{1 / 2}, \quad k \geq k_{0}
$$

Note that we can choose $k_{0} \geq \alpha$. For reader's convenience, we will estimate $\Delta \xi_{k}$ stepwise. 
Step 1 . We show that there exist $k_{1} \geq k_{0}$ and $\Gamma>0$ such that

$$
\frac{\gamma^{k}}{\alpha} \sum_{i=k}^{k+\alpha-1} s_{i}-\frac{A_{k}^{2}}{2}+\frac{1}{\alpha} \sum_{i=k}^{k+\alpha-1}\left[\frac{(i+1) \zeta_{i}^{2}}{i r_{i}-\zeta_{i}}-\frac{\zeta_{i}^{2}}{r_{i}}\right] \geq \Gamma, \quad k \geq k_{1}
$$

Applying $\zeta_{k} \in(\delta, 0)$ for $k \geq k_{0}$, we have (see (3.4) and (3.6))

$$
\begin{aligned}
\frac{\gamma^{k}}{\alpha} \sum_{i=k}^{k+\alpha-1} s_{i}-\frac{A_{k}^{2}}{2}+\frac{1}{\alpha} \sum_{i=k}^{k+\alpha-1}\left[\frac{(i+1) \zeta_{i}^{2}}{i r_{i}-\zeta_{i}}-\frac{\zeta_{i}^{2}}{r_{i}}\right] \\
=\frac{r^{k+\alpha-1}}{\alpha} \sum_{i=k}^{k} s_{i}-\frac{1}{2}\left(\frac{2}{\alpha} \sum_{i=k}^{k+\alpha-1} \frac{1}{r_{i}}\right)^{-1}+\frac{1}{\alpha} \sum_{i=k}^{k+\alpha-1}\left[\frac{\zeta_{i}^{2} r_{i}+\zeta_{i}^{3}}{r_{i}\left(i r_{i}-\zeta_{i}\right)}\right] \\
\geq \frac{\gamma^{k+\alpha-1}}{\alpha} \sum_{i=k}^{k} s_{i}-\frac{1}{4}\left(\frac{1}{\alpha} \sum_{i=k}^{k+\alpha-1} \frac{1}{r_{i}}\right)^{-1}-\frac{1}{\alpha} \sum_{i=k}^{k+\alpha-1} \delta^{2} \frac{r^{+}-\delta}{r^{-}\left(i r^{-}\right)} \\
\geq \\
\quad\left(\frac{1}{\alpha} \sum_{i=k}^{k+\alpha-1} s_{i}\right)\left[\gamma-\frac{1}{4}\left(\frac{1}{\alpha} \sum_{i=k}^{k+\alpha-1} \frac{1}{r_{i}}\right)^{-1}\left(\frac{1}{\alpha} \sum_{i=k}^{k+\alpha-1} s_{i}\right)^{-1}\right] \\
\quad-\frac{1}{\alpha k} \sum_{i=k}^{k+\alpha-1} \frac{\delta^{2}\left(r^{+}-\delta\right)}{\left(r^{-}\right)^{2}}>\vartheta(r-K)-\frac{1}{k}\left(\frac{\delta^{2}\left(r^{+}-\delta\right)}{\left(r^{-}\right)^{2}}\right), \quad k \geq k_{0} .
\end{aligned}
$$

Thus, there exist $\Gamma>0$ and $k_{1}$ with the property that (3.21) is satisfied for all $k \geq k_{1}$.

Step 2. It holds (see (3.17) and (3.20))

$$
\xi_{k}+\frac{A_{k}^{2}}{2}+\frac{B_{k}^{2}}{2}=\frac{A_{k}^{2}}{2}-\left|\xi_{k}\right|+\frac{B_{k}^{2}}{2}=\frac{1}{2}\left(A_{k}-B_{k}\right)^{2} \geq 0, \quad k \geq k_{0} .
$$

Step 3 . We prove that there exists $k_{2} \geq k_{1}$ satisfying

$$
\frac{1}{\alpha} \sum_{i=k}^{k+\alpha-1} \frac{\zeta_{i}^{2}}{r_{i}}-\frac{B_{k}^{2}}{2} \geq-\frac{\Gamma}{2}, \quad k \geq k_{2}
$$

where $\Gamma$ is taken from Step 1. Considering (3.16), we obtain

$$
\left|\zeta_{m}-\zeta_{n}\right| \leq \sum_{i=k}^{k+\alpha-1}\left|\Delta \zeta_{i}\right|<\sum_{i=k}^{k+\alpha-1} \frac{r s^{+} r^{-}-\delta\left(r^{+}-\delta\right)}{i r^{-}} \leq \frac{1}{k} \sum_{i=k}^{k+\alpha-1} \frac{r s^{+} r^{-}-\delta\left(r^{+}-\delta\right)}{r^{-}}
$$

for each $m, n \in\{k, \ldots, k+\alpha-1\}, k \geq k_{0}$. Thus, it is true

$$
\left|\zeta_{m}-\zeta_{n}\right|<\frac{D}{k}, \quad m, n \in\{k, \ldots, k+\alpha-1\}, k \geq k_{0}
$$


where

$$
D:=\alpha \frac{r s^{+} r^{-}-\delta\left(r^{+}-\delta\right)}{r^{-}}
$$

Now we can calculate (see (3.17))

$$
\begin{aligned}
\frac{1}{\alpha} \sum_{i=k}^{k+\alpha-1} \frac{\zeta_{i}^{2}}{r_{i}}-\frac{B_{k}^{2}}{2} & =\frac{1}{\alpha} \sum_{i=k}^{k+\alpha-1} \frac{\zeta_{i}^{2}}{r_{i}}-\frac{1}{2}\left(\frac{2}{\alpha} \sum_{i=k}^{k+\alpha-1} \frac{1}{r_{i}}\right) \xi_{k}^{2} \\
& =\frac{1}{\alpha} \sum_{i=k}^{k+\alpha-1} \frac{\zeta_{i}^{2}-\xi_{k}^{2}}{r_{i}}=-\frac{1}{\alpha} \sum_{i=k}^{k+\alpha-1} \frac{\left(\left|\xi_{k}\right|+\left|\zeta_{i}\right|\right)\left(\left|\xi_{k}\right|-\left|\zeta_{i}\right|\right)}{r_{i}} \\
& >\frac{2 \delta}{\alpha} \sum_{i=k}^{k+\alpha-1} \frac{\left|\xi_{k}-\zeta_{i}\right|}{r_{i}}>\frac{2 \delta D}{\alpha k} \sum_{i=k}^{k+\alpha-1} \frac{1}{r_{i}} \geq \frac{2 \delta D}{k r^{-}}, \quad k \geq k_{0}
\end{aligned}
$$

Let us discuss the inequality before the final one in more detail. If we denote

$$
\begin{aligned}
& \widehat{\zeta}_{-}^{k}:=\max \left\{\zeta_{j} ; \zeta_{j} \leq \xi_{k}, j \in\{k, \ldots, k+\alpha-1\}\right\}, \\
& \widehat{\zeta}_{+}^{k}:=\min \left\{\zeta_{j} ; \zeta_{j} \geq \xi_{k}, j \in\{k, \ldots, k+\alpha-1\}\right\},
\end{aligned}
$$

then we easily get (applying (3.26))

$$
\left|\zeta_{i}-\xi_{k}\right| \leq \max \left\{\left|\zeta_{i}-\widehat{\zeta}_{-}^{k}\right|,\left|\zeta_{i}-\widehat{\zeta}_{+}^{k}\right|\right\}<\frac{D}{k}, \quad i \in\{k, \ldots, k+\alpha-1\}, k \geq k_{0}
$$

Of course, (3.28) implies the existence of $k_{2} \geq k_{1}$ such that (3.24) is satisfied.

Using the previous steps, it is possible to prove the following result. If $l$ tends to infinity, then so do $\xi_{l}$. Combining (3.21), (3.23), and (3.24), we obtain

$$
\Delta \xi_{k} \geq \frac{1}{k+\alpha-1}\left(\Gamma+0-\frac{\Gamma}{2}\right)=\frac{\Gamma}{2(k+\alpha-1)}, \quad k \geq k_{2} .
$$

We use the estimate (3.18) because $\Delta \xi_{k}>0$. Summing inequality (3.31) from $k_{2}$ to an integer $(l-1) \geq k_{2}$, we have

$$
\xi_{l} \geq \xi_{k_{2}}+\frac{\Gamma}{2} \sum_{i=k_{2}}^{l-1} \frac{1}{i+\alpha-1} .
$$

This estimate implies that

$$
\liminf _{l \rightarrow \infty} \xi_{l} \geq \xi_{k_{2}}+\frac{\Gamma}{2} \sum_{i=k_{2}}^{\infty} \frac{1}{i+\alpha-1}=\infty .
$$


Particularly, $\xi_{k}>0$ for sufficiently large $k$ which means that $\zeta_{k}>0$ for infinitely many $k$. This contradiction gives that (3.1) is oscillatory for $\gamma>K$. problem

To prove the non-oscillatory part of Theorem 3.1, we will consider the initial value

$$
\Delta \zeta_{k}=\frac{1}{k}\left[\gamma s_{k}+\zeta_{k}+\frac{(k+1) \zeta_{k}^{2}}{k r_{k}-\zeta_{k}}\right], \quad \zeta_{\tilde{k}}=-\left(\frac{2}{\alpha} \sum_{i=1}^{\alpha} \frac{1}{r_{i}}\right)^{-1}
$$

for some integer

$$
\tilde{k}>\bar{k}\left(-\left(\frac{2}{\alpha} \sum_{i=1}^{\alpha} \frac{1}{r_{i}}\right)^{-1}, \alpha\right)
$$

where $\bar{k}$ satisfies (3.14) and (3.15). Let $\gamma<K$. Analogously as in the first part of the proof, we put

$$
\xi_{\tilde{k}}:=\frac{1}{\alpha} \sum_{i=\tilde{k}}^{\tilde{k}+\alpha-1} \zeta_{i}<0
$$

and we express (for $\left.\Delta \xi_{\tilde{k}}>0\right)$

$$
\begin{aligned}
\Delta \xi_{\tilde{k}}=\frac{1}{\alpha} \sum_{i=\tilde{k}}^{\tilde{k}+\alpha-1} \Delta \zeta_{i}=\frac{1}{\alpha} \sum_{i=\tilde{k}}^{\tilde{k}+\alpha-1} \frac{1}{i}\left[\gamma s_{i}+\zeta_{i}+\frac{(i+1) \zeta_{i}^{2}}{i r_{i}-\zeta_{i}}\right] \\
\leq \frac{1}{\tilde{k}}\left[\frac{\gamma^{\tilde{k}+\alpha-1}}{\alpha} \sum_{i=\tilde{k}} s_{i}+\xi_{\tilde{k}}+\frac{1}{\alpha} \sum_{i=\tilde{k}}^{\tilde{k}+\alpha-1} \frac{(i+1) \zeta_{i}^{2}}{i r_{i}-\zeta_{i}}\right] \\
=\frac{1}{\tilde{k}}\left[\frac{\gamma}{\alpha} \sum_{i=\tilde{k}}^{\tilde{k}+\alpha-1} s_{i}-\frac{1}{4}\left(\frac{1}{\alpha} \sum_{i=1}^{\alpha} \frac{1}{r_{i}}\right)^{-1}+\frac{1}{4}\left(\frac{1}{\alpha} \sum_{i=1}^{\alpha} \frac{1}{r_{i}}\right)^{-1}\right. \\
\left.+\xi_{\tilde{k}}+\frac{1}{\alpha} \sum_{i=\tilde{k}}^{\tilde{k}+\alpha-1} \frac{\zeta_{i}^{2}}{r_{i}}-\frac{1}{\alpha} \sum_{i=\tilde{k}}^{\tilde{k}+\alpha-1} \frac{\zeta_{i}^{2}}{r_{i}}+\frac{1}{\alpha} \sum_{i=\tilde{k}}^{\tilde{k}+\alpha-1} \frac{(i+1) \zeta_{i}^{2}}{i r_{i}-\zeta_{i}}\right]
\end{aligned}
$$

or $\left(\right.$ for $\left.\Delta \xi_{\widetilde{k}}<0\right)$

$$
\begin{aligned}
& \Delta \xi_{\tilde{k}}=\frac{1}{\alpha} \sum_{i=\tilde{k}}^{\widetilde{k}+\alpha-1} \Delta \zeta_{i} \leq \frac{1}{\widetilde{k}+\alpha-1}\left[\frac{\gamma}{\alpha} \sum_{i=\widetilde{k}}^{\tilde{k}+\alpha-1} s_{i}-\frac{1}{4}\left(\frac{1}{\alpha} \sum_{i=1}^{\alpha} \frac{1}{r_{i}}\right)^{-1}+\frac{1}{4}\left(\frac{1}{\alpha} \sum_{i=1}^{\alpha} \frac{1}{r_{i}}\right)^{-1}\right. \\
& \left.+\xi_{\tilde{k}}+\frac{1}{\alpha} \sum_{i=\tilde{k}}^{\tilde{k}+\alpha-1} \frac{\zeta_{i}^{2}}{r_{i}}-\frac{1}{\alpha} \sum_{i=\tilde{k}}^{\tilde{k}+\alpha-1} \frac{\zeta_{i}^{2}}{r_{i}}+\frac{1}{\alpha} \sum_{i=\tilde{k}}^{\tilde{k}+\alpha-1} \frac{(i+1) \zeta_{i}^{2}}{i r_{i}-\zeta_{i}}\right] .
\end{aligned}
$$


Again, we estimate $\Delta \xi_{\tilde{k}}$ stepwise. Using (3.14) and

$$
-r^{+}<-\frac{r^{+}}{2} \leq-\left(\frac{2}{\alpha} \sum_{i=1}^{\alpha} \frac{1}{r_{i}}\right)^{-1}
$$

we have

$$
\zeta_{k}>-2 r^{+}, \quad k \geq \tilde{k}, \quad \zeta_{\tilde{k}}, \ldots, \zeta_{\tilde{k}+\alpha-1}<0 .
$$

Similarly to the first part of the proof, we can show that

$$
\begin{aligned}
& \frac{\gamma^{\tilde{k}}}{\alpha} \sum_{i=\tilde{k}}^{\tilde{+} \alpha-1} s_{i}-\frac{1}{4}\left(\frac{1}{\alpha} \sum_{i=1}^{\alpha} \frac{1}{r_{i}}\right)^{-1}+\frac{1}{\alpha} \sum_{i=\tilde{k}}^{\tilde{k}+\alpha-1} \frac{(i+1) \zeta_{i}^{2}}{i r_{i}-\zeta_{i}}-\frac{1}{\alpha} \sum_{i=\tilde{k}}^{\tilde{k}+\alpha-1} \frac{\zeta_{i}^{2}}{r_{i}} \\
& =\left(\frac{1}{\alpha} \sum_{i=\tilde{k}}^{\tilde{k}+\alpha-1} s_{i}\right)\left[\gamma-\frac{1}{4}\left(\frac{1}{\alpha} \sum_{i=1}^{\alpha} \frac{1}{r_{i}}\right)^{-1}\left(\frac{1}{\alpha} \sum_{i=\tilde{k}}^{\tilde{k}+\alpha-1} s_{i}\right)^{-1}\right]+\frac{1}{\alpha} \sum_{i=\tilde{k}}^{\tilde{k}+\alpha-1} \frac{r_{i} \zeta_{i}^{2}+\zeta_{i}^{3}}{i r_{i}^{2}-\zeta_{i} r_{i}} \\
& \leq\left(\frac{1}{\alpha} \sum_{i=\tilde{k}}^{\tilde{k}+\alpha-1} s_{i}\right) \frac{r-K}{2}+\frac{1}{\alpha} \sum_{i=\tilde{k}}^{\tilde{k}+\alpha-1} \frac{r^{+}\left(2 r^{+}\right)^{2}+\left(2 r^{+}\right)^{3}}{i\left(r^{-}\right)^{2}} \\
& \leq\left(\frac{1}{\alpha} \sum_{i=\tilde{k}}^{\tilde{k}+\alpha-1} s_{i}\right) \frac{\gamma-K}{2}+\frac{12\left(r^{+}\right)^{3}}{\widetilde{k}\left(r^{-}\right)^{2}}
\end{aligned}
$$

Thus (consider (3.4)), there exist $\widehat{k} \in \mathbb{N}$ and

$$
\widehat{\Gamma}>\frac{K-\gamma}{2} \vartheta
$$

such that

$$
\frac{\gamma^{\tilde{k}+\alpha-1}}{\alpha} \sum_{i=\tilde{k}} s_{i}-\frac{1}{4}\left(\frac{1}{\alpha} \sum_{i=1}^{\alpha} \frac{1}{r_{i}}\right)^{-1}+\frac{1}{\alpha} \sum_{i=\tilde{k}}^{\tilde{k}+\alpha-1} \frac{(i+1) \zeta_{i}^{2}}{i r_{i}-\zeta_{i}}-\frac{1}{\alpha} \sum_{i=\tilde{k}}^{\tilde{k}+\alpha-1} \frac{\zeta_{i}^{2}}{r_{i}} \leq-\widehat{\Gamma}
$$

for $\tilde{k} \geq \widehat{k}$. Henceforth, let $\tilde{k} \geq \widehat{k}$.

Now we want to estimate

$$
Y:=\frac{1}{4}\left(\frac{1}{\alpha} \sum_{i=1}^{\alpha} \frac{1}{r_{i}}\right)^{-1}+\xi_{\tilde{k}}+\frac{1}{\alpha} \sum_{i=\tilde{k}}^{\tilde{k}+\alpha-1} \frac{\zeta_{i}^{2}}{r_{i}}
$$


Firstly, consider that, for $\zeta_{i}=\zeta_{\widetilde{k}}, i \in\{\tilde{k}, \ldots, \tilde{k}+\alpha-1\}$, it is valid

$$
Y=\frac{1}{4}\left(\frac{1}{\alpha} \sum_{i=1}^{\alpha} \frac{1}{r_{i}}\right)^{-1}-\left(\frac{2}{\alpha} \sum_{i=1}^{\alpha} \frac{1}{r_{i}}\right)^{-1}+\left(\frac{2}{\alpha} \sum_{i=1}^{\alpha} \frac{1}{r_{i}}\right)^{-2} \frac{1}{\alpha} \sum_{i=\tilde{k}}^{\tilde{k}+\alpha-1} \frac{1}{r_{i}}
$$

and hence (see (3.5) and (3.42))

$$
|Y-0|=\left|\left(\frac{2}{\alpha} \sum_{i=1}^{\alpha} \frac{1}{r_{i}}\right)^{-2}\left[\frac{1}{\alpha} \sum_{i=\tilde{k}}^{\tilde{k}+\alpha-1} \frac{1}{r_{i}}-\frac{1}{\alpha} \sum_{i=1}^{\alpha} \frac{1}{r_{i}}\right]\right|<\frac{1}{8}(K-\gamma) \vartheta<\frac{\widehat{\Gamma}}{4}
$$

because

$$
\frac{1}{4}\left(\frac{1}{\alpha} \sum_{i=1}^{\alpha} \frac{1}{r_{i}}\right)^{-1}-\left(\frac{2}{\alpha} \sum_{i=1}^{\alpha} \frac{1}{r_{i}}\right)^{-1}+\left(\frac{2}{\alpha} \sum_{i=1}^{\alpha} \frac{1}{r_{i}}\right)^{-2} \frac{1}{\alpha} \sum_{i=1}^{\alpha} \frac{1}{r_{i}}=0
$$

We repeat that (see (3.15))

$$
\left|\zeta_{\tilde{k}}-\zeta_{\tilde{k}+j}\right|<\frac{\Theta}{\widetilde{k}}, \quad j \in\{1, \ldots, \alpha-1\},
$$

which gives

$$
\left|\zeta_{\tilde{k}}-\xi_{\tilde{k}}\right|<\frac{\Theta}{\widetilde{k}}
$$

Considering (3.40), (3.46), (3.48), and (3.49) together for general $Y$, we have

$$
\begin{aligned}
|Y| & \leq \frac{\widehat{\Gamma}}{4}+\left|\xi_{\tilde{k}}-\zeta_{\tilde{k}}\right|+\frac{1}{\alpha} \sum_{i=0}^{\alpha-1} \frac{\left|\zeta_{\tilde{k}+i}^{2}-\zeta_{\tilde{k}}^{2}\right|}{r_{\tilde{k}+i}} \\
& =\frac{\widehat{\Gamma}}{4}+\left|\xi_{\tilde{k}}-\zeta_{\tilde{k}}\right|+\frac{1}{\alpha} \sum_{i=0}^{\alpha-1} \frac{\left|\zeta_{\tilde{k}+i}+\zeta_{\tilde{k}}\right| \cdot\left|\zeta_{\tilde{k}+i}-\zeta_{\tilde{k}}\right|}{r_{\tilde{k}+i}}<\frac{\widehat{\Gamma}}{4}+\left(1+\frac{4 r^{+}}{r^{-}}\right) \frac{\Theta}{\widetilde{k}}
\end{aligned}
$$

Since it suffices to consider very large $\tilde{k}$, we can assume that $|Y|<2 \widehat{\Gamma} / 3$.

Altogether, we obtain

$$
\Delta \xi_{\tilde{k}} \leq \frac{1}{\widetilde{k}+\alpha-1}\left(-\widehat{\Gamma}+\frac{2 \widehat{\Gamma}}{3}\right)<0 .
$$


The resulting inequality (3.51) implies

$$
\Delta \xi_{\tilde{k}}=\frac{1}{\alpha} \sum_{i=\tilde{k}}^{\tilde{k}+\alpha-1} \Delta \zeta_{i}=\frac{\zeta_{\tilde{k}+\alpha}-\zeta_{\tilde{k}}}{\alpha}<0 \text {, i.e., } \zeta_{\tilde{k}+\alpha}<\zeta_{\tilde{k}}
$$

Partially, if

$$
\zeta_{k}=-\left(\frac{2}{\alpha} \sum_{i=1}^{\alpha} \frac{1}{r_{i}}\right)^{-1} \quad \text { for some } k \geq \tilde{k}
$$

then from (3.14) it follows

$$
\zeta_{k}, \zeta_{k+1}, \ldots, \zeta_{k+\alpha}<0, \quad \zeta_{k+\alpha}<\zeta_{k}
$$

In fact (see the below given), this result remains true also if

$$
\zeta_{k} \in\left(-\eta-\left(\frac{2}{\alpha} \sum_{i=1}^{\alpha} \frac{1}{r_{i}}\right)^{-1},-\left(\frac{2}{\alpha} \sum_{i=1}^{\alpha} \frac{1}{r_{i}}\right)^{-1}\right)
$$

for a number $\eta>0$ which depends only on $\gamma$ and $K$. Considering (3.15) for large $\bar{k}$, it is seen that the solution of the Cauchy problem (3.34) satisfies

$$
\zeta_{\tilde{k}}, \zeta_{\tilde{k}+\alpha^{\prime}} \ldots, \zeta_{\tilde{k}+n \alpha^{\prime}} \ldots<-\left(\frac{2}{\alpha} \sum_{i=1}^{\alpha} \frac{1}{r_{i}}\right)^{-1}
$$

and hence (see (3.54))

$$
\zeta_{\tilde{k}+n}<0, \quad n \in \mathbb{N}
$$

Therefore, (3.57) and Lemma 2.9 say that (3.1) is non-oscillatory for $\gamma<K$.

It means that, to complete the proof, it suffices to find $\eta>0$ which guaranties the above-mentioned generalization, that is, we need to prove (3.51) for (3.55) with $k=\tilde{k}$. The concrete initial value was not used in the proof of (3.43). Thus, $\widehat{\Gamma}$ depends only on $\gamma$ and $K$. Let

$$
\eta:=\min \left\{\left(\frac{2}{\alpha} \sum_{i=1}^{\alpha} \frac{1}{r_{i}}\right)^{-1},\left(1+\frac{3 r^{+}}{r^{-}}\right)^{-1} \widehat{\Gamma} \frac{}{4}\right\}
$$


In the estimate of $Y$, since (3.14) and (3.15) remain true, we have (consider also (3.39), (3.48), and (3.49))

$$
\begin{gathered}
\zeta_{\tilde{k}+j} \in\left(-2 r^{+}, 0\right), \\
\left|\zeta_{\tilde{k}+j}+\left(\frac{2}{\alpha} \sum_{i=1}^{\alpha} \frac{1}{r_{i}}\right)^{-1}\right|<\frac{\Theta}{\widetilde{k}}+\eta, \quad\left|\xi_{\tilde{k}}+\left(\frac{2}{\alpha} \sum_{i=1}^{\alpha} \frac{1}{r_{i}}\right)^{-1}\right|<\frac{\Theta}{\widetilde{k}}+\eta,
\end{gathered}
$$

where $j \in\{0,1, \ldots, \alpha-1\}$, and (see again (3.39))

$$
\begin{aligned}
|Y| & \leq \frac{\widehat{\Gamma}}{4}+\left|\xi_{\tilde{k}}+\left(\frac{2}{\alpha} \sum_{i=1}^{\alpha} \frac{1}{r_{i}}\right)^{-1}\right|+\frac{1}{\alpha} \sum_{j=0}^{\alpha-1}\left[\frac{1}{r_{\tilde{k}+j}}\left|\zeta_{\tilde{k}+j}^{2}-\left(\frac{2}{\alpha} \sum_{i=1}^{\alpha} \frac{1}{r_{i}}\right)^{-2}\right|\right] \\
& <\frac{\widehat{\Gamma}}{4}+\frac{\Theta}{\widetilde{k}}+\eta+\frac{1}{\alpha} \sum_{j=0}^{\alpha-1}\left[\frac{1}{r_{\tilde{k}+j}}\left|\zeta_{\tilde{k}+j}-\left(\frac{2}{\alpha} \sum_{i=1}^{\alpha} \frac{1}{r_{i}}\right)^{-1}\right| \cdot\left|\zeta_{\tilde{k}+j}+\left(\frac{2}{\alpha} \sum_{i=1}^{\alpha} \frac{1}{r_{i}}\right)^{-1}\right|\right] \\
& <\frac{\widehat{\Gamma}}{4}+\frac{\Theta}{\widetilde{k}}+\eta+\frac{1}{r^{-}}\left(\frac{\Theta}{\tilde{k}}+\eta\right)\left(2 r^{+}+r^{+}\right) \\
& =\frac{\widehat{\Gamma}}{4}+\left[1+\frac{3 r^{+}}{r^{-}}\right]\left(\frac{\Theta}{\tilde{k}}+\eta\right) \leq\left[1+\frac{3 r^{+}}{r^{-}}\right] \frac{\Theta}{\tilde{k}}+\frac{\widehat{\Gamma}}{2},
\end{aligned}
$$

which confirms $|Y|<2 \widehat{\Gamma} / 3$ and then the validity of (3.51).

Remark 3.2. Let us point out that the constant $K$ arises from the calculations in Step 1.

Remark 3.3. If $r_{k}=s_{k}=1, k \in \mathbb{N}$, then $K=1 / 4$ (see (3.3)); that is, Theorem 3.1 reduces to the result about the discrete Euler equation.

Example 3.4. For arbitrarily given continuous function $f:[-1,1] \rightarrow \mathbb{R}^{+}$and $a>1, b, c \in \mathbb{R}$, let us consider

$$
\Delta\left(\frac{\Delta x_{k}}{a+\sin (b k) \cos (c k)}\right)+\frac{r f(\sin k)}{k(k+1)} x_{k+1}=0, \quad k \in \mathbb{N}
$$

The almost periodicity of $\left\{r_{k}\right\}=\left\{[a+\sin (b k) \cos (c k)]^{-1}\right\}_{k \in \mathbb{N}}$ and $\left\{s_{k}\right\}=\{f(\sin k)\}_{k \in \mathbb{N}}$ follows from Corollary 2.4 and from, for example, [45, Theorem 1.27] and [47, Theorem 1.9]. It is seen that

$$
M\left(\left\{r_{k}^{-1}\right\}\right)=a, \quad M\left(\left\{s_{k}\right\}\right)=\frac{1}{2 \pi} \int_{-\pi}^{\pi} f(\sin x) d x
$$

Thus, (3.61) is oscillatory if

$$
\gamma>K=\frac{\pi}{2 a \int_{-\pi}^{\pi} f(\sin x) d x}
$$

and non-oscillatory if $\gamma<K$. 
Analogously, under the additional condition $b \neq 0$, the oscillation constant for the equation

$$
\Delta\left([a+\sin (b k) \cos (b k)] \Delta x_{k}\right)+\frac{\gamma f(\cos k)}{k(k+1)} x_{k+1}=0, \quad k \in \mathbb{N},
$$

is

$$
K=\frac{\pi^{2}}{b \int_{-\pi}^{\pi} f(\cos x) d x \int_{-\pi / b}^{\pi / b} d y /(a+\sin (b y) \cos (b y))}=\frac{\pi \sqrt{4 a^{2}-1}}{4 \int_{-\pi}^{\pi} f(\cos x) d x}
$$

Evidently, any periodic sequence is almost periodic. Thus, we also obtain this new result.

Corollary 3.5. The equation

$$
\Delta\left(r_{k} \Delta x_{k}\right)+\frac{\gamma s_{k}}{(k+1) k} x_{k+1}=0, \quad k \in \mathbb{N},
$$

where $\gamma \in \mathbb{R}$ and $\left\{r_{k}\right\}$ and $\left\{s_{k}\right\}$ are positive sequences with period $n \in \mathbb{N}$, is oscillatory if

$$
\gamma>K:=\left[\left(\frac{2}{n} \sum_{i=1}^{n} \frac{1}{r_{i}}\right)\left(\frac{2}{n} \sum_{i=1}^{n} s_{i}\right)\right]^{-1},
$$

and non-oscillatory if $\gamma<K$.

Remark 3.6. The border case given by $\gamma=K$ remains open. Nevertheless, based on the corresponding continuous case (see [23]) and other cases which generalize the discrete equation with constant coefficients (see, e.g., [10] with references cited therein), we conjecture that (3.66) (with periodic coefficients) is non-oscillatory even for $\gamma=K$.

Example 3.7. Let an odd integer $m \geq 3$ be given. We can use Corollary 3.5 for the equation

$$
\Delta\left(\left|\sin \frac{(2 k-1) \pi}{12}\right| \Delta x_{k}\right)+\frac{r}{k(k+1)}\left(1+\cos \frac{2 k \pi}{m}\right) x_{k+1}=0, \quad k \in \mathbb{N} .
$$

Since we can choose $n=6 m$, we obtain

$$
\begin{gathered}
M\left(\left\{r_{k}^{-1}\right\}\right)=\frac{1}{6 m} \sum_{i=1}^{6 m} \frac{1}{r_{i}}=\frac{1}{6} \sum_{i=1}^{6}\left|\sin \frac{(2 i-1) \pi}{12}\right|^{-1}=\frac{\sqrt{2}}{3}(1+2 \sqrt{3}), \\
M\left(\left\{s_{k}\right\}\right)=\frac{1}{6 m} \sum_{i=1}^{6 m} s_{i}=\frac{1}{m} \sum_{i=1}^{m}\left(1+\cos \frac{2 i \pi}{m}\right)=1 .
\end{gathered}
$$


Hence, the oscillation constant for (3.68) is

$$
K=\frac{3}{4 \sqrt{2}(1+2 \sqrt{3})} \approx \frac{2}{17} .
$$

We add that we can use Theorem 3.1 also in the case when one of the sequences $\left\{r_{k}\right\}$ and $\left\{s_{k}\right\}$ in (3.1) changes its sign. If the sequence $\left\{r_{k}\right\}$ in (3.71) changes its sign, then we have to generalize the definition of the generalized zeros as follows. An interval $(a, a+1], a \in \mathbb{N}$, contains the generalized zero of a solution $\left\{x_{k}\right\}$ of (3.71) if $x_{a} \neq 0$ and $r_{a} x_{a} x_{a+1} \leq 0$.

Corollary 3.8. Let the equation

$$
\Delta\left(r_{k} \Delta x_{k}\right)+\frac{\gamma s_{k}}{(k+1) k} x_{k+1}=0, \quad k \in \mathbb{N},
$$

where $\gamma \in \mathbb{R}$ and $\left\{r_{k}\right\}$ and $\left\{s_{k}\right\}$ are nonzero almost periodic sequences, be given.

(i) If $\inf \left\{r_{k} ; k \in \mathbb{N}\right\}>0$ and $\gamma<\left[4 M\left(\left\{r_{k}^{-1}\right\}\right) M\left(\left\{\left|s_{k}\right|\right\}\right)\right]^{-1}$, then (3.71) is non-oscillatory.

(ii) If $\inf \left\{\left|r_{k}\right| ; k \in \mathbb{N}\right\}>0,\left\{s_{k}\right\}$ is positive and $\gamma>\left[4 M\left(\left\{\left|r_{k}\right|^{-1}\right\}\right) M\left(\left\{s_{k}\right\}\right)\right]^{-1}$, then (3.71) is oscillatory.

Proof. Since the almost periodicity of $\left\{f_{k}\right\}$ implies the almost periodicity of $\left\{\left|f_{k}\right|\right\}$, it suffices to apply the discrete Sturm comparison theorem and Theorem 3.1.

At the end we remark that it is possible to find several definitions of almost periodicity for $k \in \mathbb{N}$ in the literature. For example, concerning almost periodic sequences with indices $k \in \mathbb{N}$, we refer to [50]. There is proved that, for any precompact sequence $\left\{x_{k}\right\}_{k \in \mathbb{N}}$, there exists a permutation $P$ of the set of positive integers such that the sequence $\left\{x_{P(k)}\right\}_{k \in \mathbb{N}}$ is almost periodic. In fact, the so-called asymptotically almost periodic sequences are considered in [50] (based on the Bochner concept), where a bounded sequence $\left\{x_{k}\right\}_{k \in \mathbb{N}}$ is called asymptotically almost periodic if the set of sequences $\left\{x_{k+p}\right\}_{k \in \mathbb{N}}, p \in \mathbb{N}$, is precompact in the space of all bounded sequences. We add that a sequence $\left\{x_{k}\right\}_{k \in \mathbb{N}}$ is asymptotically almost periodic if and only if it is the sum of an almost periodic sequence and a sequence which approaches zero as $k \rightarrow \infty$. One finds that this representation is unique. See, for example, [51, 52].

We consider difference equations with almost periodic coefficients given by the limitation of almost periodic sequences on $\mathbb{Z}$ because this approach is the standard one. But we conjecture that the main result can be similarly proved for almost periodic coefficients defined in other ways (e.g., for the above-mentioned asymptotically almost periodic sequences).

\section{Acknowledgments}

The authors thank the anonymous referees for their suggestions and references which improved the final version of the paper. This research is supported by the Czech Science Foundation under Grant P201/10/1032. 


\section{References}

[1] S. Z. Chen and L. H. Erbe, "Riccati techniques and discrete oscillations," Journal of Mathematical Analysis and Applications, vol. 142, no. 2, pp. 468-487, 1989.

[2] J. W. Hooker and W. T. Patula, "Riccati type transformations for second-order linear difference equations," Journal of Mathematical Analysis and Applications, vol. 82, no. 2, pp. 451-462, 1981.

[3] M. K. Kwong, J. W. Hooker, and W. T. Patula, "Riccati type transformations for second-order linear difference equations, II," Journal of Mathematical Analysis and Applications, vol. 107, no. 1, pp. 182-196, 1985.

[4] Z. Došlá and Š. Pechancová, "Conjugacy and phases for second order linear difference equation," Computers E Mathematics with Applications, vol. 53, no. 7, pp. 1129-1139, 2007.

[5] O. Došlý and Š. Pechancová, "Generalized zeros of $2 \times 2$ symplectic difference system and of its reciprocal system," Advances in Difference Equations, vol. 2011, Article ID 571935, 23 pages, 2011.

[6] S. Z. Chen and L. H. Erbe, "Oscillation and nonoscillation for systems of self-adjoint second-order difference equations," SIAM Journal on Mathematical Analysis, vol. 20, no. 4, pp. 939-949, 1989.

[7] R. Koplatadze, G. Kvinikadze, and I. P. Stavroulakis, "Oscillation of second-order linear difference equations with deviating arguments," Advances in Mathematical Sciences and Applications, vol. 12, no. 1, pp. 217-226, 2002.

[8] S. H. Saker and S. S. Cheng, "Kamenev type oscillation criteria for nonlinear difference equations," Czechoslovak Mathematical Journal, vol. 54, no. 4, pp. 955-967, 2004.

[9] O. Došlý and R. Hilscher, "A class of Sturm-Liouville difference equations: (non)oscillation constants and property BD," Computers \& Mathematics with Applications, vol. 45, no. 6-9, pp. 961-981, 2003.

[10] S. Fišnarová, "Oscillation of two-term Sturm-Liouville difference equations," International Journal of Difference Equations, vol. 1, no. 1, pp. 81-99, 2006.

[11] P. Hasil, "Conjugacy of self-adjoint difference equations of even order," Abstract and Applied Analysis, vol. 2011, Article ID 814962, 16 pages, 2011.

[12] O. Došly, "Constants in the oscillation theory of higher order Sturm-Liouville differential equations," Electronic Journal of Differential Equations, vol. 2002, no. 34, pp. 1-12, 2002.

[13] L. Erbe and S. Hilger, "Sturmian theory on measure chains," Differential Equations and Dynamical Systems, vol. 1, no. 3, pp. 223-244, 1993.

[14] L. Erbe, "Oscillation results for second-order linear equations on a time scale," Journal of Difference Equations and Applications, vol. 8, no. 11, pp. 1061-1071, 2002.

[15] B. Jia, "Wong's comparison theorem for second order linear dynamic equations on time scales," Journal of Mathematical Analysis and Applications, vol. 349, no. 2, pp. 556-567, 2009.

[16] L. Erbe, J. Baoguo, and A. Peterson, "Oscillation and nonoscillation of solutions of second order linear dynamic equations with integrable coefficients on time scales," Applied Mathematics and Computation, vol. 215, no. 5, pp. 1868-1885, 2009.

[17] D. Willett, "On the oscillatory behavior of the solutions of second order linear differential equations," Annales Polonici Mathematici, vol. 21, pp. 175-194, 1969.

[18] J. S. W. Wong, "Oscillation and nonoscillation of solutions of second order linear differential equations with integrable coefficients," Transactions of the American Mathematical Society, vol. 144, pp. 197-215, 1969.

[19] B. G. Zhang and Y. Zhou, "Comparison theorems and oscillation criteria for difference equations," Journal of Mathematical Analysis and Applications, vol. 247, no. 2, pp. 397-409, 2000.

[20] L. H. Erbe and B. G. Zhang, "Oscillation of discrete analogues of delay equations," Differential and Integral Equations, vol. 2, no. 3, pp. 300-309, 1989.

[21] A. Kneser, “Untersuchungen über die reellen Nullstellen der Integrale linearer Differentialgleichungen," Mathematische Annalen, vol. 42, no. 3, pp. 409-435, 1893.

[22] F. Gesztesy and M. Ünal, "Perturbative oscillation criteria and Hardy-type inequalities," Mathematische Nachrichten, vol. 189, pp. 121-144, 1998.

[23] K. M. Schmidt, "Critical coupling constants and eigenvalue asymptotics of perturbed periodic SturmLiouville operators," Communications in Mathematical Physics, vol. 211, no. 2, pp. 465-485, 2000.

[24] O. Došlý and P. Hasil, "Critical oscillation constant for half-linear differential equations with periodic coefficients," Annali di Matematica Pura ed Applicata, vol. 190, no. 3, pp. 395-408, 2011.

[25] P. Hasil, "Conditional oscillation of half-linear differential equations with periodic coefficients," Archivum Mathematicum, vol. 44, no. 2, pp. 119-131, 2008.

[26] H. Krüger, "On perturbations of quasiperiodic Schrödinger operators," Journal of Differential Equations, vol. 249, no. 6, pp. 1305-1321, 2010. 
[27] H. Krüger and G. Teschl, "Effective Prüfer angles and relative oscillation criteria," Journal of Differential Equations, vol. 245, no. 12, pp. 3823-3848, 2008.

[28] H. Krüger and G. Teschl, "Relative oscillation theory for Sturm-Liouville operators extended," Journal of Functional Analysis, vol. 254, no. 6, pp. 1702-1720, 2008.

[29] H. Krüger and G. Teschl, "Relative oscillation theory, weighted zeros of the Wronskian, and the spectral shift function," Communications in Mathematical Physics, vol. 287, no. 2, pp. 613-640, 2009.

[30] K. Ammann and G. Teschl, "Relative oscillation theory for Jacobi matrices," in Difference Equations and Applications, pp. 105-115, Uğur-Bahçeşehir University Publications, Istanbul, Turkey, 2009.

[31] P. B. Naĭman, "The set of isolated points of increase of the spectral function pertaining to a limitconstant Jacobi matrix," Izvestiya Vysshikh Uchebnykh Zavedenii. Matematika, vol. 1959, no. 1 (8), pp. 129-135, 1959 (Russian).

[32] F. Luef and G. Teschl, "On the finiteness of the number of eigenvalues of Jacobi operators below the essential spectrum," Journal of Difference Equations and Applications, vol. 10, no. 3, pp. 299-307, 2004.

[33] J. Yu, Z. Guo, and X. Zou, "Periodic solutions of second order self-adjoint difference equations," Journal of the London Mathematical Society, vol. 71, no. 1, pp. 146-160, 2005.

[34] Z. Guo and J. Yu, "Existence of periodic and subharmonic solutions for second-order superlinear difference equations," Science in China A, vol. 46, no. 4, pp. 506-515, 2003.

[35] Z. Guo and J. Yu, "The existence of periodic and subharmonic solutions of subquadratic second order difference equations," Journal of the London Mathematical Society, vol. 68, no. 2, pp. 419-430, 2003.

[36] H. J. Li and C. C. Yeh, "An oscillation criterion of almost-periodic Sturm-Liouville equations," The Rocky Mountain Journal of Mathematics, vol. 25, no. 4, pp. 1417-1429, 1995.

[37] P. Rehák, "Comparison theorems and strong oscillation in the half-linear discrete oscillation theory," The Rocky Mountain Journal of Mathematics, vol. 33, no. 1, pp. 333-352, 2003.

[38] O. Došlý and P. Řehák, "Recessive solution of half-linear second order difference equations," Journal of Difference Equations and Applications, vol. 9, no. 1, pp. 49-61, 2003.

[39] H. A. El-Morshedy, "Oscillation and nonoscillation criteria for half-linear second order difference equations," Dynamic Systems and Applications, vol. 15, no. 3-4, pp. 429-450, 2006.

[40] P. Řehák, "A critical oscillation constant as a variable of time scales for half-linear dynamic equations," Mathematica Slovaca, vol. 60, no. 2, pp. 237-256, 2010.

[41] P. Řehák, "On certain comparison theorems for half-linear dynamic equations on time scales," Abstract and Applied Analysis, vol. 2004, no. 7, pp. 551-565, 2004.

[42] P. Ǩehák, "New results on critical oscillation constants depending on a graininess," Dynamic Systems and Applications, vol. 19, no. 2, pp. 271-287, 2010.

[43] R. P. Agarwal, Difference Equations and Inequalities: Theory, Methods, and Applications, Marcel Dekker, New York, NY, USA, 2000.

[44] W. G. Kelley and A. C. Peterson, Difference Equations: An Introduction with Applications, Academic Press, San Diego, Calif, USA, 2001.

[45] C. Corduneanu, Almost Periodic Functions, John Wiley \& Sons, New York, NY, USA, 1968.

[46] C. Corduneanu, Almost Periodic Oscillations and Waves, Springer, New York, NY, USA, 2009.

[47] A. M. Fink, Almost Periodic Differential Equations, Springer, Berlin, Germany, 1974.

[48] M. Veselý, "Almost periodic sequences and functions with given values," Archivum Mathematicum, vol. 47, no. 1, pp. 1-16, 2011.

[49] K. M. Schmidt, "Oscillation of the perturbed Hill equation and the lower spectrum of radially periodic Schrödinger operators in the plane," Proceedings of the American Mathematical Society, vol. 127, no. 8, pp. 2367-2374, 1999.

[50] R. Jajte, “On almost-periodic sequences," Colloquium Mathematicum, vol. 13, pp. 265-267, 1964-1965.

[51] W. M. Ruess and W. H. Summers, "Minimal sets of almost periodic motions," Mathematische Annalen, vol. 276, no. 1, pp. 145-158, 1986.

[52] H. L. Yao, "Some results for asymptotically almost periodic functions and asymptotically almost periodic sequences," Heilongjiang Daxue Ziran Kexue Xuebao, vol. 23, no. 6, pp. 794-796, 2006. 


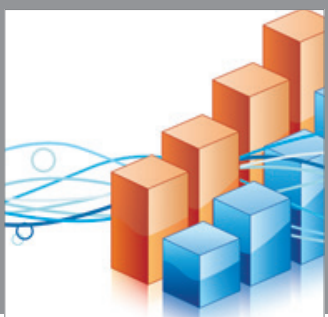

Advances in

Operations Research

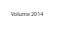

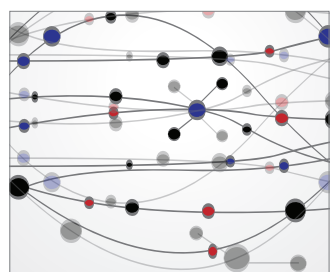

\section{The Scientific} World Journal
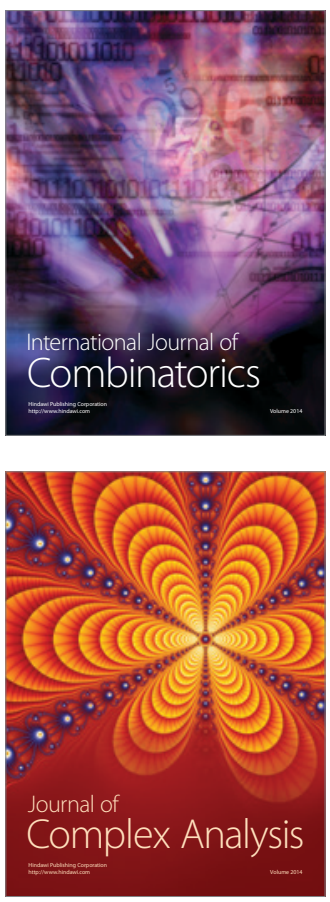

International Journal of

Mathematics and

Mathematical

Sciences
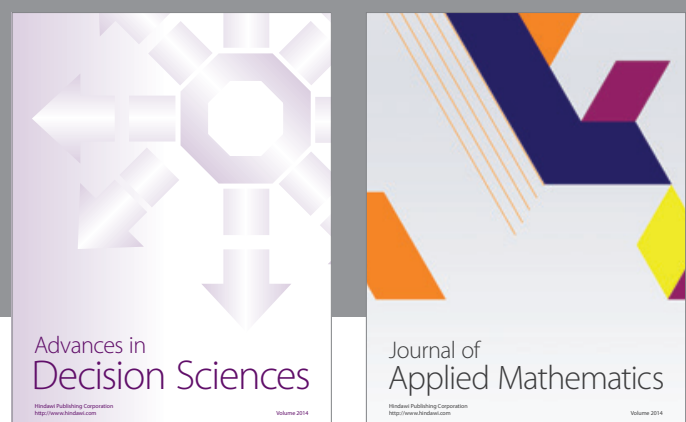

Journal of

Applied Mathematics
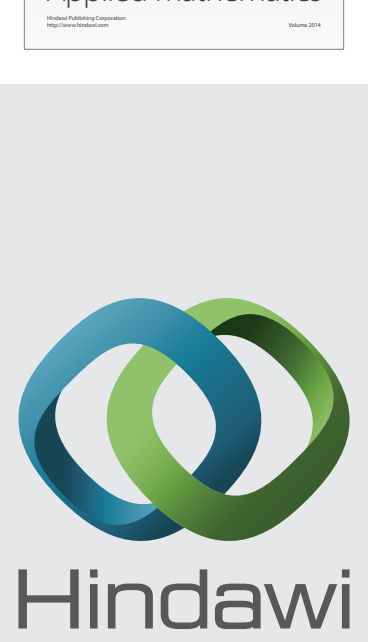

Submit your manuscripts at http://www.hindawi.com
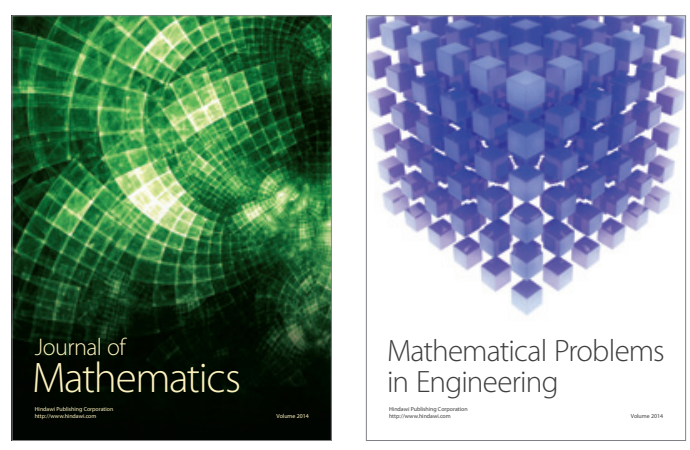

Mathematical Problems in Engineering
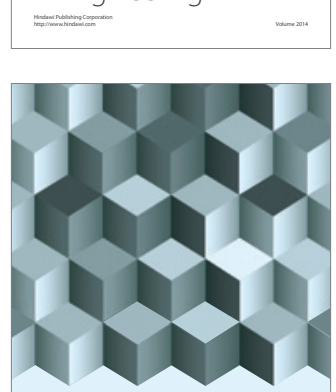

Journal of

Function Spaces
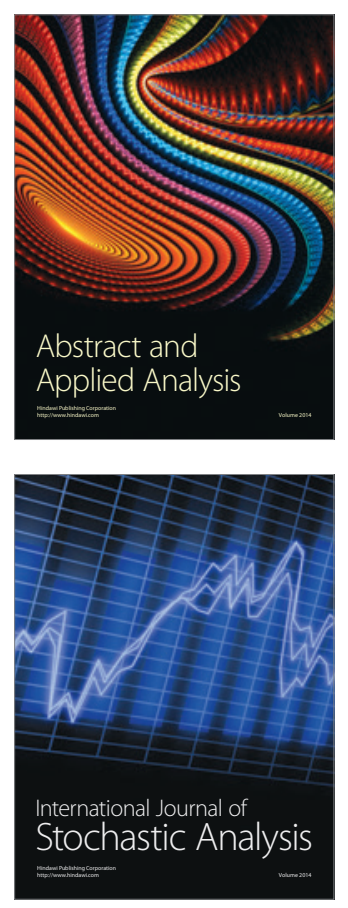

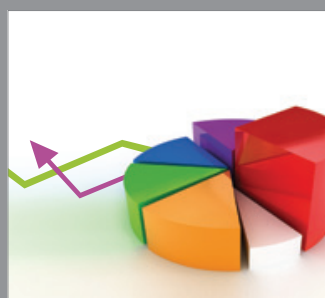

ournal of

Probability and Statistics

Promensencen
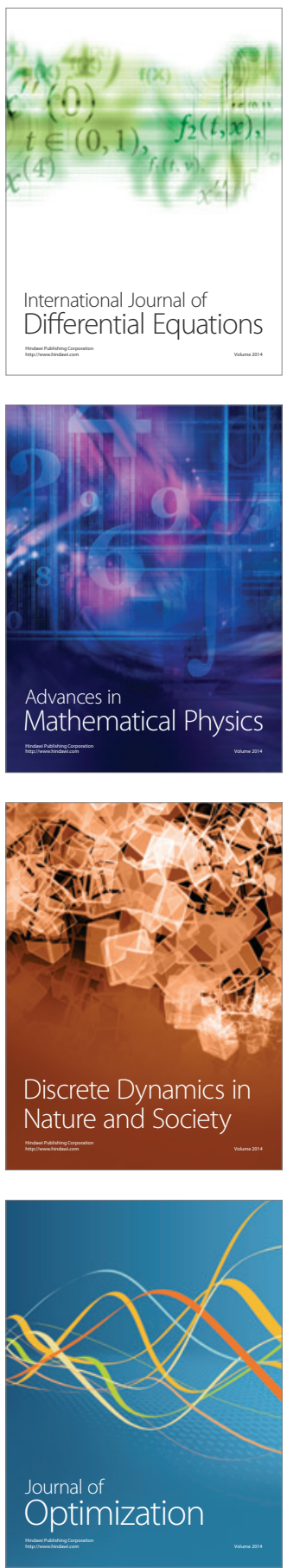